\title{
DERMATOMIOSITE CANINA-SÍMILE em cão sem raça definida: relato de caso
}

\section{Canine dermatomyositis-like disease in a mixed-breed dog: case report}

\author{
Paolo Ruggero Errante ${ }^{1,2, *}$; $;$ Priscyla Taboada Dias da Silva ${ }^{3}$; Yara Cláudia Vasconcelos ${ }^{1}$ \\ *Autor Correspondente: Paolo Ruggero Errante. Rua Pedro de Toledo, 699, 7 andar, Vila \\ Mariana, São Paulo, SP, Brasil, CEP 04039-032.E-mail: errantepr@yahoo.com
}

\begin{abstract}
Como citar: ERRANTE, Paolo Ruggero; SILVA, Priscyla Taboada Dias da; VASCONCELOS, Yara Cláudia. Dermatomiosite canina-símile em cão sem raça definida: relato de caso. Revista de Educação Continuada em Medicina Veterinária e Zootecnia do CRMV-SP, São Paulo, v.18, n. 2, 2020. Doi. 10.36440/recmvz. $\underline{\mathrm{v} 18 \mathrm{i} 2.38068}$
\end{abstract}

Cite as: ERRANTE, Paolo Ruggero; SILVA, Priscyla Taboada Dias da; VASCONCELOS, Yara Cláudia. Canine dermatomyositis-like disease in a mixed-breed dog: case report. Journal of Continuing Education in Animal Science of CRMV-SP, São Paulo, v.18, n.2, 2020. Doi. 10.36440/recmvz.v18i2.38068

\section{Resumo}

A dermatomiosite canina é uma vasculopatia inflamatória da pele e músculos, com manifestações cutâneas envolvendo a face, orelhas, extremidades da cauda e distais por sobre proeminências ósseas. 0 envolvimento muscular manifesta-se sob a forma de mioatrofia cefálica, dificuldade de deglutição, redução do reflexo do vômito e marcha atípica. A dermatomiosite canina é classificada em dermatomiosite canina familiar e em forma variante ou dermatomiosite-símile. Neste relato descreve-se um caso de dermatomiosite-símile em cadela de dois anos de idade, sem raça definida, que apresentava necrose isquêmica dos pavilhões auriculares e atrofia dos músculos temporal e masseter. O exame dermo-histopatológico realizado em fragmentos de pele dos pavilhões auriculares, região cefálica e amostras de tecido muscular (temporal e masseter) confirmou a existência de dermatite perivascular e foliculite de interface pobre em células, focos de atrofia folicular e de fibras musculares e moderada fibrose dérmica. Portanto a histopatologia, associada às manifestações clínicas do animal, permitiu o estabelecimento do diagnóstico de dermatomiosite-símile, enfermidade pouco descrita na dermatologia veterinária brasileira.

Palavras-chave: Dermatomiosite canina familiar. Dermatomiosite-símile. Dermatopatia isquêmica. Cães.

\footnotetext{
1 Clínica Veterinária Reino Animal, São Paulo, SP, Brasil

2 Departamento de Farmacologia, Escola Paulista de Medicina (EPM), Universidade Federal de São Paulo (Unifesp), São Paulo, SP, Brasil

3 Histopet Anatomia Patológica Veterinária, São Paulo, SP, Brasil
} 


\section{Abstract}

Canine dermatomyositis is a skin and muscles inflammatory vasculopathy with cutaneous manifestations involving face, ears, tail and distal ends over bony prominences. Muscle involvement manifests itself in the form of muscles of the head, difficulty in swallowing, reduction of reflex of vomit and atypical gait. Canine dermatomyositis is classified into variant form or dermatomyositis-like. This report describes a case of dermatomyositis-like in a two-year-old female mixed-breed dog that presented ischemic necrosis of pinna and temporal and masseter muscle atrophy. In histopathological examinations performed with skin fragments of pinna, cephalic region and muscle samples (temporal and masseter), it was detected a perivascular dermatitis and folliculitis with poor cell interface, foci of follicular atrophy and muscle fibers with moderate dermal fibrosis confirming the diagnosis of dermatomyositis-like, relatively little described in brazilian veterinary dermatology.

Keywords: Familial canine dermatomyositis. Dermatomyositis-like. Ischemic skin disease. Dogs.

\section{Introdução}

A dermatomiosite canina é uma vasculopatia inflamatória da pele e músculos, que inclui as formas designadas como dermatomiosite familiar canina, com padrão de herança autossômica dominante nas raças Collie e Pastor de Shetland (WAHL et al., 2008), e a dermatomiosite-símile que acomete as raças Chow-Chow, Welsh Corgi, Pastor Alemão, Kuvasz, Schanauzer miniatura, Dachshund, Fox Terrier e Rottweiler (GROSS et al., 2005; CAMPBELL et al., 2008; BRESCIANI et al., 2014). A dermatomiosite canina familiar e a dermatomiosite-símile são clinicamente similares, e diferem de outras dermatopatias isquêmicas como a dermatopatia isquêmica generalizada pósvacinal (MORRIS, 2013). Na dermatomiosite canina os eventos desencadeadores incluem a vacinação antirrábica, infecção por parvovírus, trauma, estresse, estro, parto, lactação e exposição excessiva à luz solar (MACHADO et al., 2009; RONDELLI et al., 2011). As manifestações clínicas nos animais são estabelecidas em faixas etárias variáveis (HARGIS et al.,1986a; HARGIS et al., 1986b), e as lesões cutâneas envolvem a face, orelhas, ponta de cauda e extremidades distais por sobre proeminências ósseas (HARGIS et al., 1984; KUNKLE et al., 1985; MORRIS, 2013). 0 envolvimento muscular está associado a atrofia dos músculos da cabeça, dificuldade para beber água e para apreender, mastigar e engolir o alimento devido ao envolvimento dos músculos mastigatórios e esofágicos, além de redução do reflexo do vômito e deambulação atípica (HARGIS et al., 1984; HAUPT et al., 1985).

\section{Relato de caso}

Um animal da espécie canina, fêmea, sem raça definida, com dois anos de idade foi atendido em uma clínica privada paulistana, com histórico de rarefação pilosa, necrose isquêmica de extremidades de pavilhões e atrofia dos músculos temporal (Figura 1) e masseter, desde o primeiro ano de idade. 
Figura 1. Necrose de extremidade do pavilhão auricular esquerdo

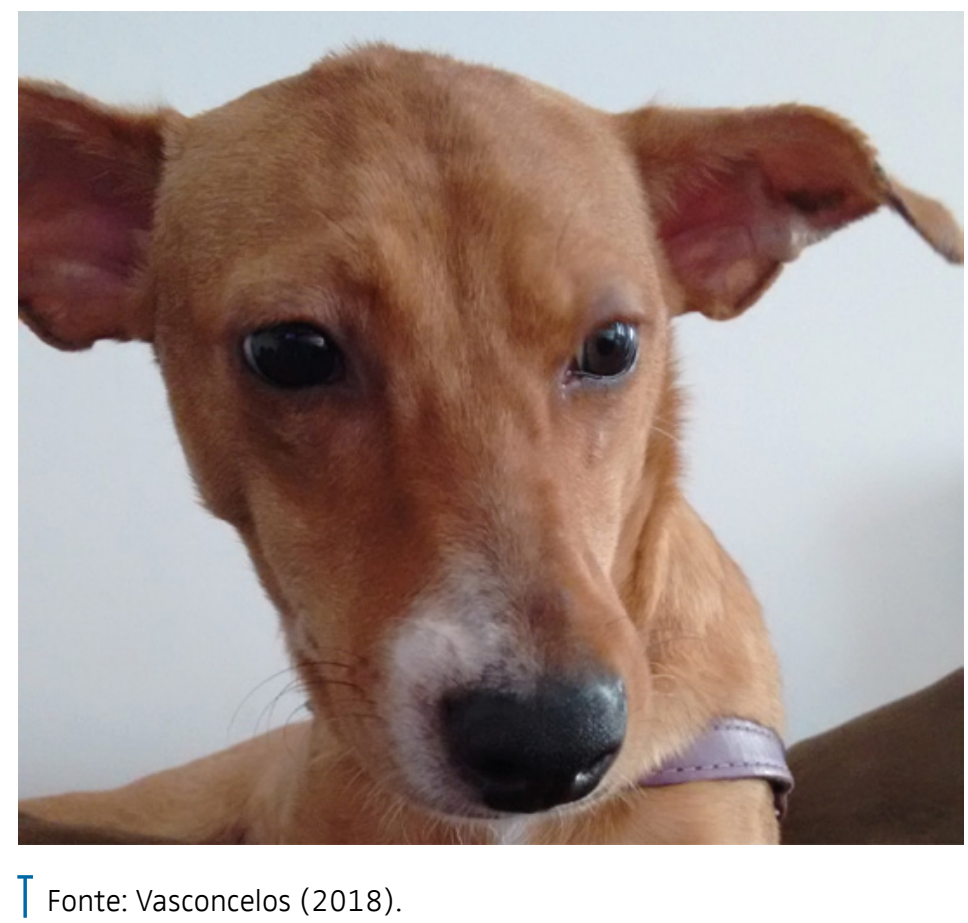

O hemograma, perfil hepático e renal, exame de urina, sorologia para leishmaniose e dermatohistopatologia foram solicitados. No exame dermatológico não foram encontrados ectoparasitas e no tricrograma os pêlos apresentavam-se em estágio telógeno. 0 raspado de pele mostrou-se negativo quanto à presença de ácaros e fungos. Quando da interposição da Lâmpada de Wood não foi evidenciada a clássica e típica fluorescência da presença de dermatófitos (Microsporum canis). Após a tricotomia, anestesia local com lidocaína 2\% sem vasoconstritor e assepsia, com uma pinça de Adson e tesoura de Matzembaum foram removidas amostras de tecido cutâneo das extremidades dos pavilhões auriculares e da região cefálica, que foram fixadas em formol tamponado a 10\%. Para a realização da biópsia do tecido muscular, o animal foi submetido a anestesia geral com indução e manutenção da anestesia com propofol (4 mg/kg) pela via intravenosa. Amostras dos músculos masseter e temporal foram obtidas por biópsia, utilizando-se de punch de Keyes $5 \mathrm{~mm}$, após realização de incisão de 1,0 cm de pele e panículo, dos músculos masseter e temporal, áreas previamente preparadas com tricotomia e antissepsia. A pele foi suturada com fio de nylon monofilamentoso 4-0 com padrão isolado simples. 0 material foi fixado em formol tamponado a $10 \%$, e a seguir as amostras foram embebidas em parafina e coradas por Hematoxilina e Eosina (H/E) (Figura 2A, B, C). O exame dermo-histopatológico foi realizado no Laboratório Histopet Anatomia Patológica Veterinária, em São Paulo, Brasil. 
Figura 2. Achados dermo-histopatológicos

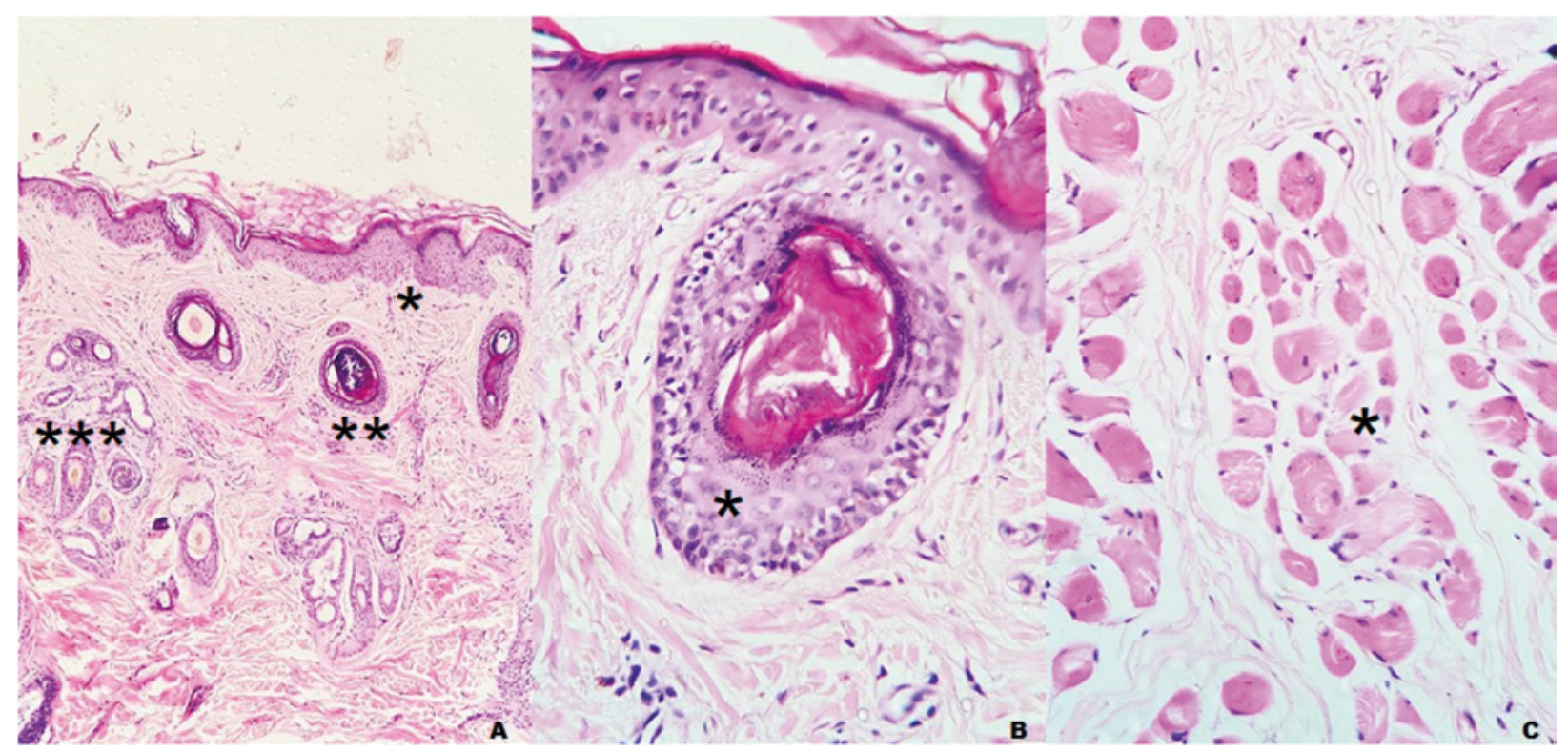

T Fonte: Silva (2018).

Legenda 2. A. Pele (orelha direita). Discreto edema da derme $\left({ }^{\star}\right)$, dermatite perivascular (**) e foliculite de interface pobre em células, atrofia folicular ( $\left.{ }^{\star \star \star}\right)$ e moderada fibrose dérmica (coloração hematoxilina/eosina, aumento 40x). B. Pele (região cefálica). Dermatite perivascular e hiperplásica, parede vascular parcialmente hialinizada (*) (coloração Hematoxilina/eosina, aumento 400x). C. Músculo temporal. Moderada fibrose e graus variados de atrofia de fibras musculares (*) (coloração hematoxilina/eosina, aumento 400x)

\section{Discussão}

As alterações microscópicas associadas as manifestações clínicas do animal foram compatíveis com o quadro clássico de dermatomiosite-símile. Tal dermatomiosite constitui-se em uma enfermidade inflamatória autoimune que acomete não só a pele, mas também a musculatura estriada esquelética e os vasos sanguíneos (MORRIS, 2013). A maioria dos casos ocorre em fêmeas, uma vez que o processo pode ser intensificado pelas alterações hormonais observadas durante o estro ou a gestação (MORRIS, 2013). Outros fatores desencadeadores da doença incluem exposição à luz solar, estresse, infecção ou aplicação de vacinas (MACHADO et al., 2009; RONDELLI et al., 2011).

A dermatomiosite canina é classificada em dermatomiosite canina familiar com padrão de herança autossômica dominante (CLARK et al., 2005; WAHL et al., 2008), ou na forma variante denominada dermatomiosite-símile (GROSS et al., 2005; BRESCIANI et al., 2014), clinicamente similares que se desenvolvem principalmente em animais jovens (MORRIS, 2013), devendo ser diferenciadas da dermatose marginal da orelha (WISSELINK, 1986), necrose trombovascular proliferativa do pavilhão auricular (MORRIS, 2013), dermatite por picada de mosca (ANGARANO, 1988), leishmaniose (CARVALHO JUNIOR et al., 2017), dermatopatia isquêmica juvenil, essa com predisposição familiar desconhecida (YOON et al., 2010), paniculite pós-aplicação de imunógeno, mormente a vacina antirrábica (HENDRICK; DUNAGAN, 1991), dermatopatia isquêmica generalizada induzida por vacinação (KIM et al., 2011), dermatopatia isquêmica em animal adulto sem associação com reação vacinal (MORRIS, 2013), demodiciose, leishmaniose, escabiose, lúpus eritematoso cutâneo, Síndrome úveo-dermatológica e dermatite alérgica (ROTHING et al., 2015).

0 animal referido no presente caso apresentou um quadro lesional tegumentar precoce, no primeiro ano de idade sob a forma de alopecia, necrose isquêmica de pavilhões auriculares e atrofia da musculatura (massetérica e temporal). Na avaliação histopatológica da pele das regiões auriculares, 
destra e sinistra, bem como, da cefálica foi observada uma degeneração vacuolar em células da camada basal, infiltrado linfocitário em parede folicular infundibular; derme e região perivascular, contendo linfócitos, plasmócitos e melanófagos. Na seção da musculatura esquelética (masseter e temporal) foi observada atrofia de fibras musculares com variação do diâmetro, achados compatíveis com a dermatite isquêmica/dermatomiosite canina (dermatomiosite-símile). 0 diagnóstico da dermatomiosite canina assenta-se no histórico clínico do animal, confirmado pelo resultado da dermato-histopatologia (HARGIS et al., 1984; HAUPT et al., 1985; GROSS; KUNKLE, 1987). Nos casos crônicos, as características histopatológicas incluem atrofia folicular, infiltração linfocitária perivascular, melanófagos e degeneração vacuolar em células da camada basal (HARGIS et al., 1984; MORRIS, 2013). Após o diagnóstico de dermatomiosite-símile, foi instituída terapia com pentoxifilina ( $25 \mathrm{mg} / \mathrm{kg} / \mathrm{Bis}$ in Die ou $B I D$ ) e prednisona ( $2 \mathrm{mg} / \mathrm{kg} / \mathrm{Semel}$ in Die ou SID) durante oito semanas com discreta melhora do quadro dermatológico. Em função desse padrão de resposta clínica, o proprietário decidiu não dar continuidade ao tratamento, optando pela eutanásia do animal. Os corticosteroides são os ativos de primeira escolha para o tratamento, assim como o derivado de xantina, com ação reológica, e a pentoxifilina (REES et al., 2003; MORRIS, 2013; ROTHING et al., 2015). Quando a enfermidade não é severa pode ser utilizada a associação tetraciclina ou doxiciclina e niacinamida pelas suas propriedades imunomodulatórias. 0 uso da ciclosporina, azatioprina e micofenolato de mofetil em combinação com corticosteroides é indicado em casos refratários (ROTHING et al., 2015). Também é indicada a vitamina E, pelas suas propriedades antioxidantes e os ácidos graxos essenciais, pelas características anti-inflamatórias (RHOUMA et al., 2013; ROTHING et al., 2015; BARROUIN-MELO, 2016). Romero et al. (2018) descreveram o caso de um animal acometido pela dermatomiosite em que houve acentuado crescimento dos pelos e melhora na qualidade da pele das unhas após oito semanas de terapia com pentoxifilina ( $25 \mathrm{mg} / \mathrm{Kg} / \mathrm{BID}$ ), doxiclina (100 mg/Kg/BID), niacinamida (500 mg/Kg/SID) e oclacitinibe $(0,4 \mathrm{mg} / \mathrm{Kg} / \mathrm{SID})$.

\section{Conclusão}

A associação entre as manifestações clínicas de um animal da espécie canina, com o resultado da dermato-histopatologia, permitiu o estabelecimento do diagnóstico da dermatomiosite-símile, enfermidade pouco descrita na dermatologia veterinária brasileira.\&

\section{Bibliografia}

ANGARANO, D. W. Diseases of the pinna. Veterinary Clinics of North America, Small Animal Practice, n. 18, p. 869-864, 1988.

BARROUIN-MELO, S. M. et al. Evaluating oxidative stress, serological and haematological status of dogs suffering from osteoarthritis, after supplementing their diet with fish or corn oil. Lipids Health Disease Journal, n. 15, p. 139, 2016.

BRESCIANI, F. et al. Dermatomyositis-like disease in a Rottweiler. Veterinary Dermatology, n.25, p. 229-232, 2014. Disponível em: https://doi.org/10.1111/vde.12128. Acesso em: 29 ago. 2020.

CAMPBELL, K. L.; LOWE, A. D.; LICHTENSTEIGER, C. A. Dermatomyositis in three Portuguese water dog littermates. Veterinary Dermatology, n. 19, p. 69, 2008.

CARVALHO JUNIOR, C. G. et al. Parasitism and inflammation in ear skin and in genital tissues of symptomatic and asymptomatic male dogs with visceral leishmaniasis. Parasitology Research, n. 116, p. 987-995, 2017. Disponível em: https://doi.org/10.1111/vde.12128 10.1007/s00436-017-53754. Acesso em: 29 ago.2020.

CLARK, L. A. et al. Linkage of dermatomyositis in the Shetland sheepdogs to chromosome 35. Veterinary Dermatology, n. 16, p. 392-394, 2005. 
GROSS, T. L.; KUNKLE, G. A. The cutaneous histology of dermatomyositis in Collie dogs. Veterinary Pathology, n. 24, p. 11-15, 1987. Disponível em: https://doi.org/10.1111/vde.1212810.1111/ j.1365-3164.2005.00469.x. Acesso em: 29 ago.2020.

GROSS, T. L. et al. Interface diseases of the dermal-epidermal junction. In: Skin diseases of the dog and the cat, California: Blackwell Science, p. 49-52, 2005.

HARGIS, A. M. et al. Familial canine dermatomyositis. Initial characterization of the cutaneous and muscular lesions. American Journal of Pathology, n. 116, p. 234-244, 1984.

HARGIS, A. M. et al. Prospective study of familial canine dermatomyositis. Correlation of the severity of dermatomyositis and circulating immune complex levels. American Journal of Pathology, n. 123, p. 465-479, 1986a.

HARGIS, A. M. et al. Postmortem findings in four litters of dogs with familial canine dermatomyositis. American Journal of Pathology, n. 123, p. 480-496, 1986b.

HAUPT, K. H. et al. Familial canine dermatomyositis: clinical, electrodiagnostic, and genetic studies. American Journal of Veterinary Research, n. 46, p. 1861-1869, 1985.

HENDRICK, M. J.; DUNAGAN, C. A. Focal necrotizing granulomatous panniculitis associated with subcutaneous injection of rabies vaccine in cats and dogs: 10 cases (1988-1989). Journal of the American Veterinary Medical Association, n. 198, p. 304-305, 1991.

$\mathrm{KIM}, \mathrm{H}$. J. et al. Long-term management of vaccine-induced refractory ischemic dermatopathy in a miniature Pinscher puppy. The Journal of Veterinary Medical Science, n. 73, p. 1237-1240, 2011. Disponível em: https://doi.org/10.1111/vde.1212810.1292/jvms.10-0524. Acesso em: 20 ago.2020.

KUNKLE, G. A. et al. Dermatomyositis in collie dogs. Compendium on Continuing Education for the Practicing Veterinarian, n. 7, p. 185-192, 1985.

MACHADO, L. H. A. et al. Dermatomiosite canina familiar: relato de caso. Clínica Veterinária, n.81, p. 106-110, 2009.

MORRIS, D. O. Ischemic dermatopathies. Veterinary Clinics of North America: Small Animal Practice, n. 43, p. 99-111, 2013. Disponível em: https://doi.org/10.1111/vde.1212810.1016/j. cvsm.2012.09.008. Acesso em: 29 ago. 2020.

REES, C. A.; BOOTHE, D. M. Therapeutic response to pentoxifylline and its active metabolite in dogs with familial canine dermatomyositis. Veterinary Therapeutics, n. 4, p. 234-241, 2003.

RHOUMA, M. et al. Anti-inflammatory response of dietary vitamin $E$ and its effects on pain and joint structures during early stages of surgically induced osteoarthritis in dogs. Canadian Journal Veterinary Research, n. 77, p. 191-198, 2013.

ROMERO, C. et al. Three cases of canine dermatomyositis-like disease. Acta Scientiae Veterinariae, $n$. 46, p.1-6, 2018.

RONDELLI, M. C. H. et al. Dermatomiosite canina: relato de três casos. Clínica Veterinária, n. 93, p. 58$62,2011$.

ROTHING, A. et al. Dermatomyositis in a Family of working Kelpies. Tierartliche Praxis. Ausgabe K, Kleintiere/Heimtiere, n. 43, p. 331-336, 2015. Disponível em: https://doi.org/10.15654/TPK-141112. Acesso em: 24 ago. 2020.

YOON, J. S. et al. Two dogs with juvenile-onset disease with involvement of extremities. The Journal of Veterinary Medical Science, n. 72, p. 1513-1516, 2010. Disponível em: https://doi.org/10.1292/ 
jvms.10-0041. Acesso em: 24 ago. 2020.

WAHL, J. M. et al. Analysis of gene transcript profiling and immunobiology in Shetland sheepdogs with dermatomyositis. Veterinary Dermatology, n. 19, p. 52-58, 2008. Disponível em: https://doi. org/10.1111/j.1365-3164.2008.00655.x. Acesso em: 24 ago. 2020.

WISSELINK, M. A. The external ear in skin diseases of dogs and cats: a diagnostic challenge. Veterinary Quarterly, n. 8, p. 318-328, 1986. Disponivel em: https://doi.org/10.1080/01652176.1986.969406 2. Acesso em 29 ago. 2020. 